\title{
Mass Generation and Non-Euclidean Metric from Fractional Dynamics
}

\author{
Ervin Goldfain \\ Research Scholar, Ronin Institute, Montclair, New Jersey 07043 \\ Email: ervin.goldfain@ronininstitute.org
}

\begin{abstract}
Fractional-time Schrödinger equation (FTSE) describes the evolution of quantum processes endowed with memory effects. FTSE manifestly breaks all consistency requirements of quantum field theory (unitarity, locality and compliance with the clustering theorem), unless the order of fractional differentiation and integration $(0<\alpha<1)$ falls close to the standard index $\alpha=1$. Working in the context of the minimal fractal manifold (where $\alpha=1-\varepsilon, \varepsilon<<1$ ), we confirm here that FTSE approximates the attributes of gravitational metric and provides an unforeseen generation mechanism for massive fields.
\end{abstract}

Key words: Fractional dynamics, fractional time Schrödinger equation, fractional field theory, minimal fractal manifold, mass generation, curvilinear coordinates, General Relativity.

Fractional-time Schrödinger equation (FTSE) assumes the form [1]

$$
i \hbar \partial_{t}^{\alpha} \psi(x, t)=H \psi(x, t)
$$

in which $\partial_{t}^{\alpha}$ is the Caputo fractional derivative, $H$ the Hamiltonian operator for $\alpha=1$ and where

$$
0<\alpha<1
$$


The infinitesimal time evolution of the wavefunction $\psi(x, t)$ may be presented as

$$
\psi(x, t+\eta)=\psi(x, t)+\frac{\eta^{\alpha}}{\Gamma(1+\alpha)}\left[-\frac{\hbar^{2}}{2 m_{\alpha}} \partial_{x}^{2}+V(x)\right] \psi(x, t)
$$

with $\eta=\Delta t<<1$. A unique feature of FTSE is the emergence of an effective dimensionless mass induced by fractional time evolution and given by [1]

$$
m_{\alpha}=\Gamma^{2}(\alpha+1)
$$

FTSE exclusively meets the consistency requirements of quantum theory in the asymptotic limit $\alpha=1-\varepsilon, \varepsilon<<1$. It is therefore natural to interpret $\varepsilon$ as the dimensional parameter of the minimal fractal manifold, according to which [2]

$$
\varepsilon=4-D=O\left(\frac{m^{2}}{\Lambda_{U V}^{2}}\right)<<1
$$

where $D$ is the spacetime dimension and $\Lambda_{U V}$ the ultraviolet energy scale. By (4), (3) and (2) may be written as, respectively,

$$
\begin{gathered}
m_{1-\varepsilon}=\Gamma^{2}(2-\varepsilon) \\
\frac{\psi(x, t+\eta)-\psi(x, t)}{\eta^{1-\varepsilon}}=\frac{1}{\Gamma(2-\varepsilon)}\left[-\frac{\hbar^{2}}{2 m_{1-\varepsilon}} \partial_{x}^{2}+V(x)\right] \psi(x, t)
\end{gathered}
$$

Accounting for (4), we further expand (5) to obtain the reasonable leading-order approximation [3]

$$
m_{1-\varepsilon} \approx 1-b_{0} \varepsilon, \quad b_{0} \neq 0
$$


Likewise, fractional series expansion of the wavefunction $\psi(x, t)$ yields [1]

$$
\frac{\psi(x, t+\eta)-\psi(x, t)}{\eta^{1-\varepsilon}}=\frac{1}{\Gamma(2-\varepsilon)} \partial_{t}^{1-\varepsilon} \psi(x, t)+O\left(\eta^{2-\varepsilon}\right)
$$

Taking the limit $\eta=\Delta t \rightarrow 0$ in (8) leads to

$$
\partial_{t} \psi(x, t)=\frac{1}{\Gamma(2-\varepsilon)} \partial_{t}^{1-\varepsilon} \psi(x, t)+O\left(\eta^{2-\varepsilon}\right)
$$

It is known that a general gravitational field cannot be made stationary in any reference frame and no conserved energy can be accordingly defined [4]. The only exception to this property is a bounded spacetime region where Lorentz invariance and energy conservation can be locally restored.

Given these observations, as well as the non-unitary nature of the FTSE, we are tempted to draw an analogy between (9) and the expression of the gradient operator in curvilinear coordinates. To this end, let $x^{\mu}(\mu=0,1,2,3)$ denote Cartesian coordinates in $D=4$ Euclidean spacetime and having the corresponding orthonormal basis $e_{v}$. Consider an orthogonal system of curvilinear coordinates $\xi^{\mu}$ in this spacetime. Invoking again the limit of nearly vanishing fractality $(\varepsilon<<1)$, the system of coordinates $\xi^{\mu}$ defines at each point a local orthogonal coordinate basis as in [5]

$$
\vec{\xi}_{\mu}=\sum_{v} e_{v} \frac{\partial x^{v}}{\partial \xi^{\mu}}
$$

Accordingly, the gradient operator defined in curvilinear coordinates reads 


$$
\vec{\nabla}=\sum_{\mu} \frac{a_{\mu}}{h_{\mu}} \frac{\partial}{\partial \xi^{\mu}}
$$

where

$$
\vec{a}_{\mu}=\frac{1}{h_{\mu}} \vec{\xi}_{\mu}
$$

Here, the metric tensor $g_{\mu \nu}=\vec{\xi}_{\mu} \cdot \vec{\xi}_{v}$ in orthogonal curvilinear coordinates has the form

$$
g_{\mu \nu}=\operatorname{diag}\left(h_{0}^{2}, h_{1}^{2}, h_{2}^{2}, h_{3}^{2}\right)
$$

with inverse

$$
g^{\rho \omega}=\operatorname{diag}\left(h_{0}^{-2}, h_{1}^{-2}, h_{2}^{-2}, h_{3}^{-2}\right)
$$

Comparative inspection of (9) and (11) suggests the following analogy between FTSE defined on the minimal fractal manifold and the non-Euclidean metric of General Relativity

$$
\frac{1}{\Gamma(2-\varepsilon)} \approx\left(1-b_{0} \varepsilon\right)^{-1 / 2} \Leftrightarrow \frac{a_{0}}{h_{0}}
$$

Summing up, (7) and (15) reveal an intriguing connection between fractional time evolution, on the one hand, the mass generation mechanism of field theory and gravitational metric, on the other. Both findings line up well with our earlier work on these topics $[2,6-8,10]$.

Can this connection pave the way towards a unified picture of fractional field theory and General Relativity from the standpoint of complex dynamics [9]? Only time will tell. 


\section{References}

1. https://www.sciencedirect.com/science/article/pii/S2590054418300010

2. Available at the following sites:

http://www.aracneeditrice.it/aracneweb/index.php/pubblicazione.html?item $=9788854$

$\underline{889972}$

https://www.researchgate.net/publication/278849474 Introduction to Fractional Fi eld Theory consolidated version

3. https://mathworld.wolfram.com/GammaFunction.html

4. Schutz, B. F. “A first course in General Relativity”, Cambridge Univ. Press, 1985.

5. Redzic, D. V., “The operator $\nabla$ in orthogonal curvilinear coordinates”, Eur. J. Phys. 22 (2001) 595-599.

6. https://www.sciencedirect.com/science/article/abs/pii/So960077905008659

7. https://www.sciencedirect.com/science/article/abs/pii/S1007570406001183

8. https://www.sciencedirect.com/science/article/abs/pii/Sog60077903004569

9. Available at the following site:

https://www.researchgate.net/publication/344227402_Complex_Dynamics_and_Fou ndational Physics

10. Available at the following site:

https://www.researchgate.net/publication/228625050 Chaotic Dynamics of the Re normalization_Group_Flow_and_Standard_Model_Parameters 
